\title{
Ultrasound-guided central venous catheter placement in the emergency department: experience in a hospital in Bogotá, Colombia
}

This article was published in the following Dove Press journal:

Open Access Emergency Medicine

\author{
German Devia Jaramillo' \\ Jenny Torres Castillo' \\ Freddy Lozano ${ }^{2}$ \\ Angélica Ramírez ${ }^{2}$
}

'Emergency Medicine Department, Universidad del Rosario, Resuscitation

Unit, Hospital Universitario

Mayor Méderi, Bogotá, Colombia;

${ }^{2}$ Resuscitation Unit, University

Hospital Mayor Méderi, Bogotá, Colombia
Correspondence: German Devia Jaramillo Emergency Medicine Department, Universidad del Rosario, Resuscitation Unit, Hospital Universitario Mayor Méderi, Bogotá, Colombia Email german.devia@urosario.edu.co
Introduction: The use of central venous catheters (CVCs) in the emergency room (ER) is a valuable tool for the comprehensive management of critically ill patients; however, the positioning of these devices is not free of complications. Currently, the use of ultrasound is considered a useful and safe tool to carry out these procedures, but in Colombia, the number of emergency departments providing this tool is scarce and there is no literature describing the experience in our country.

Objective: The objective of this study was to describe the experience regarding placement of ultrasound-guided CVCs by emergency physicians in an institution in Bogotá, as well as the associated complications.

Materials and methods: This is a descriptive cross-sectional retrospective study. Medical records of 471 patients requiring insertion of CVCs in the resuscitation area from January 2014 to December 2014 were reviewed. Insertion site and complications are described.

Results: For 471 total cases, the average age of patients was 68.6 years, the most frequent diagnosis was sepsis $(30.7 \%)$, the preferred route of insertion was the right internal jugular vein, and insertion was successful at the first attempt in $85.9 \%$ of patients. Pneumothorax was the most common complication (1.2\%), followed by extensive hematoma and infection.

Conclusion: Insertion of ultrasound-guided CVCs by emergency physicians is a safe procedure that involves complications similar to those reported in the literature; it is necessary to expand the use of ultrasound-guided CVCs in ERs.

Keywords: ultrasound, central catheter, emergency room, critical care, Colombia

\section{Introduction}

The use of central venous catheters (CVCs) in the emergency room (ER) is a valuable tool for the comprehensive management of critically ill ER patients. Indications for use of these catheters include, but are not limited to, administration of drugs, hemodynamic monitoring, quantification of perfusion rates, and insertion of temporary pacemaker sheaths and electrodes; it is well known, however, that this procedure is not innocuous and complications such as arterial puncture, pneumothorax, hemothorax, chylothorax, and hematoma may occur and multiple punctures may be needed. ${ }^{1}$ In Colombia, the use of procedural ultrasound by non-radiologist physicians is scarce due to the limited resources available and the lack of training. ${ }^{2}$ Accordingly, the use of this procedure is considered innovative in Colombia. This article is intended to describe the experience regarding placement of ultrasound-guided CVCs by emergency physicians at a tertiary university hospital in Bogotá, Colombia. 


\section{Materials and methods}

This observational study was conducted at Hospital Mayor Méderi (Bogotá, Colombia), a tertiary care university hospital in Colombia with 238,000 emergency admissions per year.

A total of 471 patients older than 18 years requiring CVCs from January 2014 to December 2014 were included; pregnant women and patients with incomplete medical records or undergoing non-ultrasound catheterization but rather using anatomic landmarks were excluded. Patients were followed up until catheter removal. To collect patient demographic and catheter insertion-related data (e.g., number of attempts, route, indications, and complications), medical records, procedural notes, and nurse notes were obtained.

\section{Ultrasound-guided method}

The insertion area was prepared and covered with a sterile drape with the patient supine. A 7.5 MHz linear-array ultrasound probe connected to a real-time ultrasound unit (SonoSite M-Turbo P08792/P09823 or Siemens Sonoline Prima Ultrasound System Ref 4900531) and focused at a depth of $6.5 \mathrm{~cm}$ was covered with ultrasonic gel and wrapped in a sterile plastic sheath. By wrapping the transducer in a sterile sheath, standard ultrasound two-dimensional (2D) imaging was used to measure the depth and caliber of the vessel and identify any sign of thrombi in the vein. Catheterization was performed under continuous dynamic observation of real-time 2D images. A $19-\mathrm{G}, 10 \mathrm{~cm}$ needle was advanced through the skin under ultrasound guidance into the vessel. A guidewire was then placed through the needle into the vein, and the needle was removed.

The statistical analysis was performed using the IBM SPSS Statistics 19 software; central tendency and dispersion measurements were used for quantitative variables, while percentages and frequencies were used for qualitative variables.

All patients who were admitted to emergency department signed a generic form (patient consent F-CME - 22 V.0). They accepted and gave us their written informed consent for the use and publication of their medical records for academic and research purposes; additionally, this research is considered a low-risk research by Colombian laws (Resolution 8430/1993).

The study design was reviewed and approved by the Hospital Research Committee.

\section{Results}

Of 596 total reviewed records, 471 met the inclusion and exclusion criteria. Among the 471 patients undergoing insertion of CVCs, 231 (49\%) were males; the mean age was 68.6 years. Indications for CVC insertion included need for continuous monitoring, 356 (75.5\%) patients; vasopressor support, $86(18.2 \%)$ patients; electrolyte replacement, eight (1.6\%) patients; metabolic monitoring, nine $(1.9 \%)$ patients; difficult peripheral access, six (1.2\%) patients; and emergency dialysis, two $(0.4 \%)$ patients (Table 1$)$. Catheter placement was achieved at the first attempt in 405 (85.9\%) patients; $53(11.2 \%)$ patients required two attempts, and 13 $(2.7 \%)$ patients required three or more attempts. Abnormal coagulation times were observed in a high percentage of patients, $212(45.0 \%)$ patients (Table 2 ).

The most common diagnoses included, but were not limited to, severe sepsis and septic shock, 145 (30.7\%) patients; decompensated diabetes mellitus, 33 (7.0\%) patients; coronary artery disease and cardiogenic shock,

Table I Patient characteristics

\begin{tabular}{ll}
\hline Variables & $\mathbf{N}=\mathbf{4 7}$ I, $\mathbf{n}(\%)$ \\
\hline $\begin{array}{l}\text { Age (years) } \\
\text { Gender }\end{array}$ & 68.6 \\
Male & $231(49)$ \\
Indication & \\
Continuous monitoring & $356(75.5)$ \\
Vasopressor support & $86(18.2)$ \\
Electrolyte replacement and metabolic monitoring & $17(3.6)$ \\
No peripheral access & $6(1.2)$ \\
Dialysis & $2(0.2)$ \\
Other & $4(0.8)$ \\
Coagulation times & \\
Abnormal & $212(45.0)$ \\
\hline
\end{tabular}

Table 2 Insertion site, position, number of attempts and complications

\begin{tabular}{ll}
\hline & $\mathbf{N}=\mathbf{4 7} \mathbf{I}, \mathbf{n}(\%)$ \\
\hline Insertion site & \\
Right internal jugular vein & $352(74.7)$ \\
Left internal jugular vein & $101(2 I .4)$ \\
Right subclavian vein & $8(1.6)$ \\
Left subclavian vein & $4(0.8)$ \\
Right femoral vein & $3(0.6)$ \\
Left femoral vein & $3(0.6)$ \\
Catheter tip position & \\
Cavoatrial junction & $337(71.5)$ \\
Superior vena cava & $6 I(12.9)$ \\
Right atrium & $72(15.2)$ \\
Ascending jugular vein & $1(0.2)$ \\
Number of attempts & \\
One & $405(85.9)$ \\
Two & $53(11.2)$ \\
Three or more & $13(2.7)$ \\
Complications & \\
Extensive hematoma & $3(0.6)$ \\
Pneumothorax & $6(1.2)$ \\
Catheter-related infection & $3(0.6)$ \\
\hline
\end{tabular}




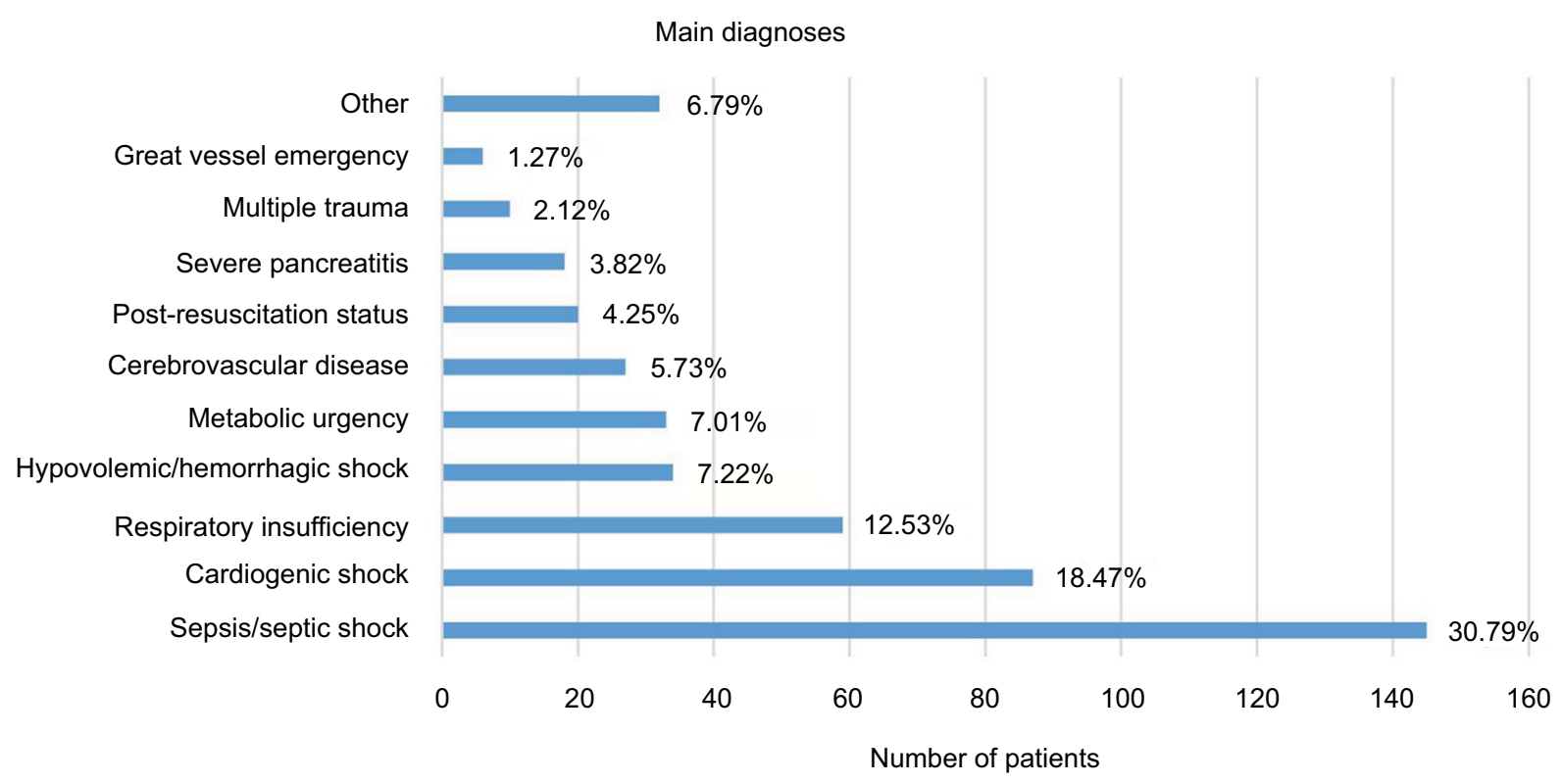

Figure I Indications for the implant of the central line.

87 (18.4\%) patients; respiratory insufficiency, 59 (12.5\%) patients; cerebrovascular disease, 27 (5.7\%) patients; and post-resuscitation status, 20 (4.2\%) patients (Figure 1).

The most common route of insertion was the right internal jugular vein, 352 (74.7\%) patients, followed by the left internal jugular vein, 101 (21.4\%) patients; the right subclavian vein, eight $(1.6 \%)$ patients; the left subclavian vein, four $(0.8 \%)$ patients; and the right and left femoral veins, three patients each $(0.6 \%)$. The rate of complications was $2.4 \%$, and included pneumothorax, six (1.2\%) patients; extensive hematoma, three $(0.6 \%)$ patients; and catheter-related infection, three $(0.6 \%)$ patients. As for placement of the catheter, $337(71.5 \%)$ remained at the cavoatrial junction, with the remaining 134 (28.4\%) placed at the superior vena cava, 61 $(12.9 \%)$; the right atrium, $72(15.2 \%)$; and the ascending jugular vein, one $(0.2 \%$; Table 2$)$.

\section{Discussion}

This study describes the experience regarding placement of ultrasound-guided CVCs by emergency physicians in a country with an emerging use of ultrasound for both clinical and procedural purposes by non-radiologist physicians. ${ }^{2}$ Based on our experience, the use of ultrasound has been shown to be very useful for the initial resuscitation of critically ill patients. Over the past few decades, the use of ultrasonography as guidance for a number of procedures such as anesthesia, intensive care, radiology, and emergency care has showed benefits for the placement of CVCs with well-established advantages $;{ }^{1,3}$ however, studies in urgency settings, especially in Latin America, are lacking. Indications to use CVCs in our study are not different from those of other studies and international guidelines, ${ }^{1,4,5}$ with the most common diagnoses being severe sepsis and septic shock and cardiovascular disease, conditions in which early monitoring of perfusion and early use of vasoactive drugs have been recommended by international guidelines. ${ }^{5,6}$ The most commonly used route of insertion was the right internal jugular vein; subclavian access was less common than reported in the literature. ${ }^{1,4}$ The catheter tip was placed at the cavoatrial junction in $71.5 \%$ of the procedures, with only one case of a catheter passing upwards distally to the target, and the remaining $28 \%$ were placed at the superior vena cava next to the atrium (12.9\%) or at the right atrium (15.2\%), locations reported as safe in the literature, with a reduced risk of stenosis or vascular thrombosis. ${ }^{7,8}$ The use of ultrasonography may help develop techniques aimed to improve proper placement of the catheter tip; ${ }^{9,10}$ however, as this study was retrospective, no real-time data regarding catheter placement could be collected.

The most common complication was pneumothorax occurring in $1.2 \%$ of patients; this is the most commonly reported complication in the literature, with rates up to $30 \%$ of the total complications during placement of central catheters; $;^{11,12}$ pneumothorax has been reported with an incidence ranging from $0 \%$ to $6.6^{13-16}$ in overall series and up to $2 \%$ with ultrasound; ${ }^{17}$ our findings are similar to those reported in the literature. Since the percentage of procedures carried 
out by personnel under training was not specified in this study, determining its influence on the results is not possible. Other complications documented in our series included hematoma $(0.6 \%)$ and infection related to the implant of the device $(0.6 \%)$. Data about minor complications such as arterial puncture were not collected from the charts that were reviewed; however, these are uncommon when ultrasoundguided procedures are used, reaching up to $1 \% .{ }^{18}$ Fatal arrhythmia, with an incidence of $0.1-0.9 \%,{ }^{19}$ or neurologic lesions were not observed, and the finding of hematoma using the ultrasonography technique is comparable to that reported in the literature, i.e., $0.5-6.1 \%,{ }^{20,21}$ it is important to notice that the population in our study showed a higher risk of bleeding as a result of age and prolonged coagulation times in $49 \%$ of cases. The low rate of infection is probably related to the appropriate aseptic techniques that were used during catheter insertion.

Most catheters were implanted after the initial attempt (85.9\% patients), two attempts were necessary in $11.2 \%$ patients, and only $2.7 \%$ patients required more than two attempts, which makes this technique guided by ultrasound a safe procedure for patients, probably allowing an appropriate use of the technique. As reported in a systematic review by Keenan ${ }^{1}$ that included 17 studies comparing the passage of catheters guided by ultrasound versus placement using anatomic landmarks, failure rates as low as 16\% $(95 \% \mathrm{CI}$ 9-23\%) and a decreased complication risk of $24 \%$ (95\% CI 8-39\%), both favoring ultrasound, were observed, without increases in procedural times. Given the retrospective nature of this study and that only patients undergoing placement of a single catheter were included, the success rate in our department is unknown; therefore, a number of prospective studies are being planned in our department to improve recording and allow a quality assessment when performing these procedures.

\section{Limitations}

Since this was a retrospective study, no additional data regarding the technique or procedural complications could be collected; additionally, the number of patients was limited, and no comparisons were performed with the usual technique of anatomic landmarks. Regardless of this, our results are comparable to those described in the literature.

Prospective studies are being planned to improve statistical power of data and, in particular, conclusively measure the benefits arising from the use of ultrasound by emergency physicians.

\section{Conclusion}

At this institution, ultrasound-guided CVC placement performed by emergency physicians has been demonstrated to be a safe procedure, as shown by the complication rate, which is similar to that described in the literature. Therefore, it remains a very useful tool for the management of critically ill patients at the ER; however, further larger prospective studies are needed, especially in this country, to strengthen our experience.

\section{Disclosure}

The authors report no conflicts of interest in this work.

\section{References}

1. Keenan SP. Use of ultrasound to place central lines. J Crit Care. 2002;17(2):126-137.

2. Henwood PC, Beversluis D, Genthon AA, et al. Characterizing the limited use of point-of-care ultrasound in Colombian emergency medicine residencies. Int J Emerg Med. 2014;7(1):7.

3. Karakitsos D, Labropoulos N, De Groot E, et al. Real-time ultrasoundguided catheterisation of the internal jugular vein: a prospective comparison with the landmark technique in critical care patients. Crit Care. 2006;10(6):R162.

4. Leung J, Duffy M, Finckh A. Real-time ultrasonographically-guided internal jugular vein catheterization in the emergency department increases success rates and reduces complications: a randomized, prospective study. Ann Emerg Med. 2006;48(5):540-547.

5. Dellinger RP, Carlet JM, Masur H, et al; Surviving Sepsis Campaign Management Guidelines Committee. Surviving Sepsis Campaign guidelines for management of severe sepsis and septic shock. Intensive Care Med. 2004;30(4):536-555.

6. Van Herck JL, Claeys MJ, De Paep R, Van Herck PL, Vrints CJ, Jorens PG. Management of cardiogenic shock complicating acute myocardial infarction. Eur Heart J Acute Cardiovasc Care. 2015;4(3):278-297.

7. Cadman A, Lawrance JA, Fitzsimmons L, Spencer-Shaw A, Swindell R. To clot or not to clot? That is the question in central venous catheters. Clin Radiol. 2004;59(4):349-355.

8. Luciani A, Clement O, Halimi P, et al. Catheter-related upper extremity deep venous thrombosis in cancer patients: a prospective study based on Doppler US. Radiology. 2001;220(3):655-660.

9. Gekle R, Dubensky L, Haddad S, et al. Saline flush test: can bedside sonography replace conventional radiography for confirmation of abovethe-diaphragm central venous catheter placement? J Ultrasound Med. 2015;34(7):1295-1299.

10. Duran-Gehring PE, Guirgis FW, McKee KC, et al. The bubble study: ultrasound confirmation of central venous catheter placement. Am J Emerg Med. 2015;33(3):315-319.

11. Mitchell SE, Clark RA. Complications of central venous catheterization. AJR Am J Roentgenol. 1979;133(3):467-476.

12. Plewa MC, Ledrick D, Sferra JJ. Delayed tension pneumothorax complicating central venous catheterization and positive pressure ventilation. Am J Emerg Med. 1995;13(5):532-535.

13. Takeyama $H$, Taniguchi $M$, Sawai $H$, et al. Limiting vein puncture to three needle passes in subclavian vein catheterization by the infraclavicular approach. Surg Today. 2006;36(9):779-782.

14. Kaiser CW, Koornick AR, Smith N, Soroff HS. Choice of route for central venous cannulation: subclavian or internal jugular vein? A prospective randomized study. J Surg Oncol. 1981;17(4):345-354.

15. Plaus WJ. Delayed pneumothorax after subclavian vein catheterization. JPEN J Parenter Enteral Nutr. 1990;14(4):414-415. 
16. Herbst CA Jr. Indications, management, and complications of percutaneous subclavian catheters. An audit. Arch Surg. 1978;113(12): 1421-1425.

17. Martin MJ, Husain FA, Piesman M, et al. Is routine ultrasound guidance for central line placement beneficial? A prospective analysis. Curr Surg. 2004;61(1):71-74.

18. Tseng M, Sadler D, Wong J, et al. Radiologic placement of central venous catheters: rates of success and immediate complications in 3412 cases. Can Assoc Radiol J. 2001;52(6):379-384.
19. Brothers TE, Von Moll LK, Niederhuber JE, Roberts JA, Walker-Andrews $\mathrm{S}$, Ensminger WD. Experience with subcutaneous infusion ports in three hundred patients. Surg Gynecol Obstet. 1988;166(4):295-301.

20. Cajozzo M, Quintini G, Cocchiera G, et al. Comparison of central venous catheterization with and without ultrasound guide. Transfus Apher Sci. 2004;31(3):199-202.

21. Chan LL, Tan BS, Kaw GJ, et al. Radiological placement of 211 central venous catheters: outcome and complications. Ann Acad Med Singapore. 1999;28(4):481-487.

\section{Publish your work in this journal}

The Open Access Emergency Medicine is an international, peerreviewed, open access journal publishing original research, reports, editorials, reviews and commentaries on all aspects of emergency medicine. The manuscript management system is completely online and includes a very quick and fair peer-review system, which is all

\section{Dovepress}

easy to use. Visit http://www.dovepress.com/testimonials.php to read real quotes from published authors. 